\title{
Bloch oscillations of ultracold atoms: a tool for a metrological determination of $\mathbf{h} / \mathbf{m}_{\mathrm{Rb}}$
}

\author{
Rémy Battesti, ${ }^{1}$ Pierre Cladé, ${ }^{1}$ Saïda Guellati-Khélifa, ${ }^{2}$ Catherine Schwob,${ }^{1}$ \\ Benoît Grémaud, ${ }^{1}$ François Nez, ${ }^{1}$ Lucile Julien, ${ }^{1}$ and François Biraben ${ }^{1}$ \\ ${ }^{1}$ Laboratoire Kastler Brossel, Ecole Normale Supérieure, \\ CNRS-UMR 8552, UPMC, 4 place Jussieu, 75252 Paris Cedex 05, France \\ ${ }^{2}$ BNM-INM, Conservatoire National des Arts et Métiers, \\ 292 rue Saint Martin, 75141 Paris Cedex 03, France
}

\begin{abstract}
We use Bloch oscillations in a horizontal moving standing wave to transfer a large number of photon recoils to atoms with a high efficiency (99.5\% per cycle). By measuring the photon recoil of ${ }^{87} R b$, using velocity selective Raman transitions to select a subrecoil velocity class and to measure the final accelerated velocity class, we have determined $h / m_{R b}$ with a relative precision of 0.4 ppm. To exploit the high momentum transfer efficiency of our method, we are developing a vertical standing wave set-up. This will allow us to measure $h / m_{R b}$ better than $10^{-8}$ and hence the fine structure constant $\alpha$ with an uncertainty close to the most accurate value coming from the $(g-2)$ determination.
\end{abstract}

PACS numbers: 32.80.Pj, 32.80.Qk, 06.20.Jr, 42.65.Dr

In the past 20 years, atom manipulation using laser light has led to the emergence of many powerful techniques [1]. In particular, it is now possible to observe and measure elementary processes between light and atoms, such as a coherent momentum transfer (absorption and emission of a single photon). Furthermore, by increasing the interrogation time, laser cooling leads to an improvement of more than two orders of magnitude in both stability and accuracy in many fields of high precision measurements [2, 3]. These advances allow us to measure precisely the recoil velocity $v_{r}$ of the atom absorbing or emitting a photon $\left(v_{r}=\hbar k / m\right.$, where $k$ is the wave vector of the photon absorbed by the atom of mass $m$ ). Such a measurement yields a determination of $h / m$ which can be used to infer a value for the fine structure constant $\alpha$ via [4]:

$$
\alpha^{2}=\frac{2 R_{\infty}}{c} \frac{M}{M_{e}} \frac{h}{m}
$$

$\mathrm{M}$ and $\mathrm{m}$ are respectively, the mass of test particle in atomic and SI Units. In this expression several terms are known with a very small uncertainty: $8 \times 10^{-12}$ for the Rydberg constant $R_{\infty}[5,6]$ and $7 \times 10^{-10}$ for the electron mass $M_{e}$ [7, 8]. A recent measurement using Penning trap single ion spectrometry allows a determination of $M_{\mathrm{Rb}}$ with an uncertainty less than $2 \times 10^{-10}[9]$. In short, the determination of $\alpha$ using this formula is now limited by the uncertainty in the ratio $h / m$ [4].

The fine structure constant can be deduced from experiments related to different branches of physics (QED, Solid state physics,...) 10, 11, 12, 13, 14, 15. . Many of these measurements lead to determinations of $\alpha$ with a relative uncertainty on the order of $10^{-8}$ but their total dispersion exceeds $10^{-7}$ (CODATA 98, 16]). In order to test the validity of these different measurements, a new accurate determination of $\alpha$ is highly desirable.

The recoil of an atom when it absorbs a photon was first observed in the recoil-induced spectral doubling of the $\mathrm{CH}_{4}$ saturated absorption peaks 17]. Since then, almost all recent measurements of the recoil velocity have been based on atomic interferometry [18] using stimulated Raman transitions between two hyperfine ground state levels [19, 20]. The precision of these experiments is increased by giving additional photon recoils to the different interferometer paths. In this process, the efficiency of the recoil transfer is a crucial parameter. Recently, S. Chu group at Stanford, using a coherent adiabatic transfer technique and high intensity Raman transitions pulses, has achieved an efficiency of $94 \%$, allowing a total momentum transfer of 120 recoils, and hence an absolute accuracy of 7.4 parts per billion in $\alpha$ 20]. D. Pritchard and colleagues have developed another tool for a determination of $h / m_{N a}$, using a Bose-Einstein condensate as a bright subrecoil atom source in the "contrast interferometry" technique 21]. This experiment seems, presently, to be limited by the low momentum transfers.

In this paper, we investigate the phenomena of Bloch oscillations of atoms driven by a constant inertial force in a periodic optical potential [23]. This method is based on stimulated Raman transitions, induced by counterpropagating laser beams, involving only one hyperfine level in order to modify the atomic momentum, thus leaving the internal state unchanged. The atoms are coherently accelerated using a frequency-chirped standing wave. In order to compensate the Doppler effect, the frequency difference between the two beams is increased linearly. Consequently, the atoms are resonant with the beams periodically. This leads to a succession of rapid adiabatic passages between momentum states differing by $2 \hbar k$ ( $2 v_{r}$ in terms of velocity). As explained above, the final accuracy is determined by the number of additional recoils, which strongly depends on the efficiency population transfer between momentum states. In our experiment, we achieve an efficiency of $99.5 \%$ per Bloch oscillation; which may provide a great opportunity for high precision 
Optical molasses

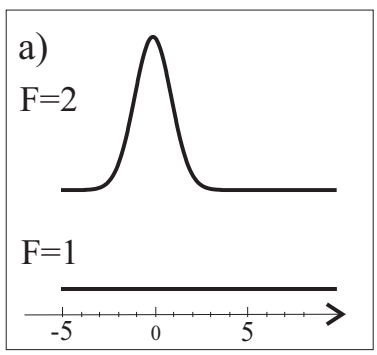

Coherent acceleration

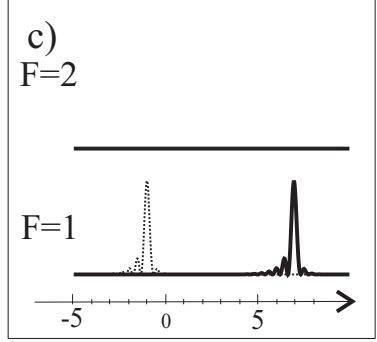

Selection

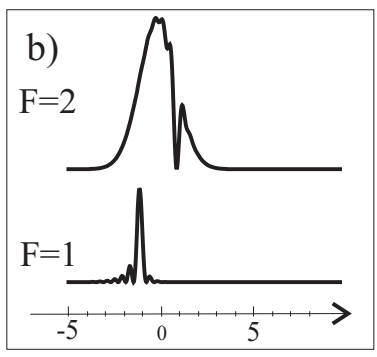

Measurement

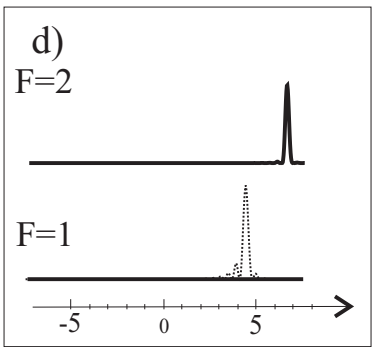

FIG. 1: Evolution of the velocity distribution (in $v_{r}$ unit) during the experiment: a) Initial velocity distribution, the atoms are in $5^{2} \mathrm{~S}_{1 / 2},\left|F=2, m_{F}=0\right\rangle$ state, b) Subrecoil selection, the atoms are transferred from $5^{2} \mathrm{~S}_{1 / 2},\left|F=2, m_{F}=0\right\rangle$ to $5^{2} \mathrm{~S}_{1 / 2},\left|F=1, m_{F}=0\right\rangle$, c) Coherent acceleration for $N=4$ Bloch oscillations, the atoms are in $5^{2} \mathrm{~S}_{1 / 2},\left|F=1, m_{F}=0\right\rangle$ and d) Measurement of the final velocity class, the atoms are transferred from $5^{2} \mathrm{~S}_{1 / 2},\left|F=1, m_{F}=0\right\rangle$ to $5^{2} \mathrm{~S}_{1 / 2}$, $\left|F=2, m_{F}=0\right\rangle$

measurement of the recoil shift.

The details of the experiment have been described previously [24]. The set-up for the laser cooling uses a magneto-optical trap (MOT) in a Rubidium vapor cell. After a few seconds, the MOT is loaded and then the magnetic field is switched off, leaving the atoms to equilibrate in an optical molasses, at a temperature of about $3 \mu \mathrm{K}$. The sequence then involves three steps (see Fig 1): first we select a narrow subrecoil velocity class with a well defined mean initial velocity using a Raman velocityselective $\pi$-pulse [25]. Next we transfer $2 N$ photon recoils by a coherent acceleration of atoms (Bloch oscillations). Finally, we measure the final velocity class, using another Raman velocity-selective $\pi$-pulse.

For the initial selection and the final measurement, the two Raman beams are generated by two Master Oscillatory Power Amplifiers (MOPAs) injected by two gratingstabilized extended-cavity laser diodes (ECLs). One of the two diodes is frequency stabilized on a highly stable Zerodur Fabry-Perot Cavity (ZFPC). This cavity was calibrated using different optical references, allowing a determination of the laser frequency with an accuracy better than $10 \mathrm{MHz}\left(3 \times 10^{-8}\right)$. A heterodyne signal and a frequency chain around the Rubidium hyperfine split-

ting $(6.8 \mathrm{GHz})$ are used to phase lock the second ECL to the first one. All auxiliary sources in the frequency chain are referenced to the same stable $10 \mathrm{MHz}$ quartz oscillator. To reduce spontaneous emission and light shift, the ECLs are detuned by about $340 \mathrm{GHz}$ from the D2 line. The MOPA beams are sent through two $80 \mathrm{MHz}$ acousto-optic modulators for timing and intensity control. Their radio frequencies are also referenced to the $10 \mathrm{MHz}$ quartz oscillator. The two beams are coupled in an optical fiber, they have linear orthogonal polarizations and their intensities are actively stabilized.

In order to perform the selection phase, we use a square Raman pulse with a frequency initially fixed at $\delta_{\text {select }}$. For a detuning of $340 \mathrm{GHz}$ and an intensity of $120 \mathrm{~mW} / \mathrm{cm}^{2}$, the $\pi$ condition is achieved using a $T=1.7 \mathrm{~ms}$ pulse. Such a pulse transfers atoms from state $5^{2} \mathrm{~S}_{1 / 2},\left|F=2, m_{F}=0\right\rangle$ to $5^{2} \mathrm{~S}_{1 / 2},\left|F=1, m_{F}=0\right\rangle$, with a velocity dispersion of about $v_{r} / 30$ centered around $\left(\lambda \delta_{\text {select }} / 2\right)-v_{r}$ where $\lambda$ is the laser wavelength. In this horizontal geometry, the width of the transferred velocity class, which is proportional to $1 / T$, is only limited by the fall of the atoms through the lasers beams. The value of $T$ represents a good compromise between resolution (the width of the selected velocity distribution) and the signal-to-noise ratio (proportional to the number of selected atoms).

After the Raman selection process, a beam resonant with $5^{2} \mathrm{~S}_{1 / 2}, F=2$ to $5^{2} \mathrm{P}_{3 / 2}, F=3$ cycling transition pushes away atoms remaining in state $F=2$. The selected atoms are then exposed to two counterpropagating beams generated by a Ti-Sapphire laser whose frequency is also stabilized to the ZFPC. This laser beam is split in two, each beam passing through an acoustooptic modulator to control its frequency. In order to perform a coherent acceleration, we vary the frequency difference between the two Bloch beams linearly with time: $\Delta \nu(t)=2 a t / \lambda$ where $a$ is the effective acceleration. The two beams are superimposed onto the horizontal optical axis of the selection Raman beams using the same optical fibers. The two Bloch beams have the same linear polarization, equal intensity (160 $\mathrm{mW}$ for each beam) and are red detuned by $100 \mathrm{GHz}$ from the $5^{2} \mathrm{~S}_{1 / 2}$ to $5^{2} \mathrm{P}_{3 / 2}$ resonance line. The duration of the acceleration process is typically $4.4 \mathrm{~ms}$. The optical potential is adiabatically turned on in about $300 \mu s$.

In the case where the constant inertial force seen by the atoms is weak enough, all the selected atoms are accelerated. In a Bloch oscillation scheme, this is equivalent to avoiding interband transitions. This condition may be expressed in the weak binding limit [26] by:

$$
\pi \frac{d \Delta \nu(t)}{d t}<<\left(\frac{U_{0}}{2 \hbar}\right)^{2}
$$

where $U_{0}$ is the depth of the potential induced by the light shift due to the standing wave. In this limit, the 
interband transition rate per Bloch period is given by a Landau-Zener formula $R=e^{\left(-a_{c} / a\right)}$ where $a_{c}$ is the critical acceleration, proportional to $\left(U_{0} / E_{r}\right)^{2}\left(E_{r}\right.$ is the recoil energy) 23. In our experiment $U_{0}$ is about $11 E_{r}$ and atoms acceleration is $133 \mathrm{~ms}^{-2}$ leading to a theoretical efficiency of $99.9 \%$ per oscillation.

After the acceleration process we perform the final velocity measurement using another Raman $\pi$-pulse, whose frequency is $\delta_{\text {measure }}$. Population transfer from the hyperfine state $\mathrm{F}=1$ to the hyperfine state $\mathrm{F}=2$ due to the second Raman pulse is maximal when $2 \pi\left(\delta_{\text {select }}-\delta_{\text {measure }}\right)=$ $2 N \times\left(\mathbf{k}_{\mathbf{1}}-\mathbf{k}_{\mathbf{2}}\right) \cdot \mathbf{k}_{\text {Bloch }} \hbar / m_{R b}$, where $\mathbf{k}_{\mathbf{1}}, \mathbf{k}_{\mathbf{2}}$ and $\mathbf{k}_{\text {Bloch }}$ are respectively the wave vectors of the Raman and Bloch beams. The populations $(F=1$ and $F=2$ ) are measured separately by using the one-dimensional time of flight technique developed for atomic clocks and depicted in 27]. The detection zone is $15 \mathrm{~cm}$ below the center of the trap (Figure 21). To avoid the horizontal motion of the atoms, and in order for the atoms to reach the detection zone, a symmetric acceleration-deceleration scheme is used : instead of selecting atoms at rest, we first accelerate them to $2 N v_{r}$, using $\mathrm{N}$ Bloch oscillations, then we make the three steps sequence: selection, coherent deceleration ( $N$ Bloch oscillations) and measurement, according to Fig. 11.

Fig. 2p shows a typical time of flight signal for $F=2$ (left peak) and $F=1$ (right peak) when the second Raman frequency is centered at the top of the final velocity distribution. In this figure, we present the signal for $N=0$ and for $N=40$ Bloch oscillations. Comparing the number of atoms between the two situations, we demonstrate that the losses during the Bloch acceleration for $N=40$ are less than $20 \%$, corresponding to a transfer efficiency of about $99.5 \%$ per oscillation. These losses are not due to the spontaneous emission, which is evaluated to $0.1 \%$ per oscillation, but probably to the residual horizontal displacement of the atoms (about $5 \mathrm{~mm}$ ) after the acceleration-deceleration process.

To reduce systematic errors, we perform an alternate and symmetric recoil transfer in both horizontal opposite directions. We determine the recoil frequency by a differential measurement of the center of the two final velocity distributions. Fig 3 shows a typical scan of final velocity distribution for $N=50$ Bloch oscillations for both directions. Each of the 400 data points corresponds to a single cycle (cooling, selection, acceleration of $2 N v_{r}$, and measure). From a data analysis of 200 points (10 minutes) we can split the final velocity distribution with an uncertainty of $v_{r} / 5000$. Hence, the relative uncertainty of the measurement of $v_{r}$ is $1.5 \times 10^{-6}$.

Fig. 4 shows, chronologically, 43 determinations of $h / m_{R b}$ using such measurements, compared to the expected value of $h / m_{R b}$, using the CODATA 98 value of $\alpha$. The mean value lies $6.1 \times 10^{-7}$ above the expected value with a relative uncertainty of $4.2 \times 10^{-7}$. We have estimated errors form wave front curvature (1.3 ppb), dif-
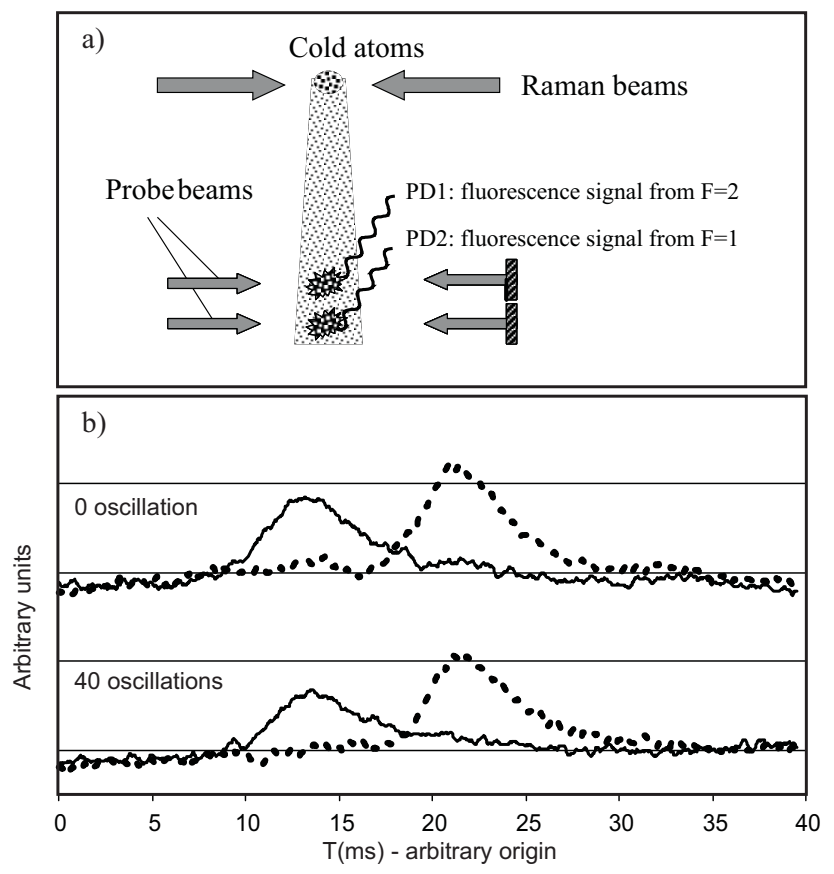

FIG. 2: a) Experimental scheme. b) Time of flight signal for $N=0$ and $N=40$ Bloch oscillations. The dashed line corresponds to the signal of the $F=1$ atoms and the solid line for the $F=2$ atoms which are transferred from the hyperfine state $F=1$ by the second Raman pulse. After 40 Bloch oscillations the time of flight signal remains almost unchanged, emphasizing thus the high efficiency transfer of our experiment. The analysis of these signals gives the number of atoms in each hyperfine state.

ferential light shift ( $80 \mathrm{ppb})$, Zeeman effect ( $57 \mathrm{ppb}$ ) and laser frequencies $(52 \mathrm{ppb})$. They are an order of magnitude lower compared to this disagreement. We believe that this disagreement and the dispersion of the results ( $\chi^{2}=99$ for 43 measurements) can be explained by the systematic variations of the initial velocity distribution of the cold cloud and the phase fluctuation of the Raman beams. These effects have not yet been quantified.

In conclusion, we have demonstrated that coherent acceleration using Bloch oscillations is a powerful method to transfer a large number of additional photon recoils to atoms. In the horizontal scheme, the number of momentum recoil transfers is limited by the fall of atoms. In order to take advantage of the high transfer efficiency of the Bloch oscillation technique, we plan to build a set-up with vertical Bloch and Raman beams. In this case, the number of additional recoils will be limited by the transverse motion, and we can increase the $\pi$-pulse duration in order to select a narrower velocity class. Moreover, due to the gravitational acceleration $g$, the vertical motion is more complicated and this geometry provides scope for two different experiments: either the atoms are accelerated by a moving standing wave as in the horizontal 

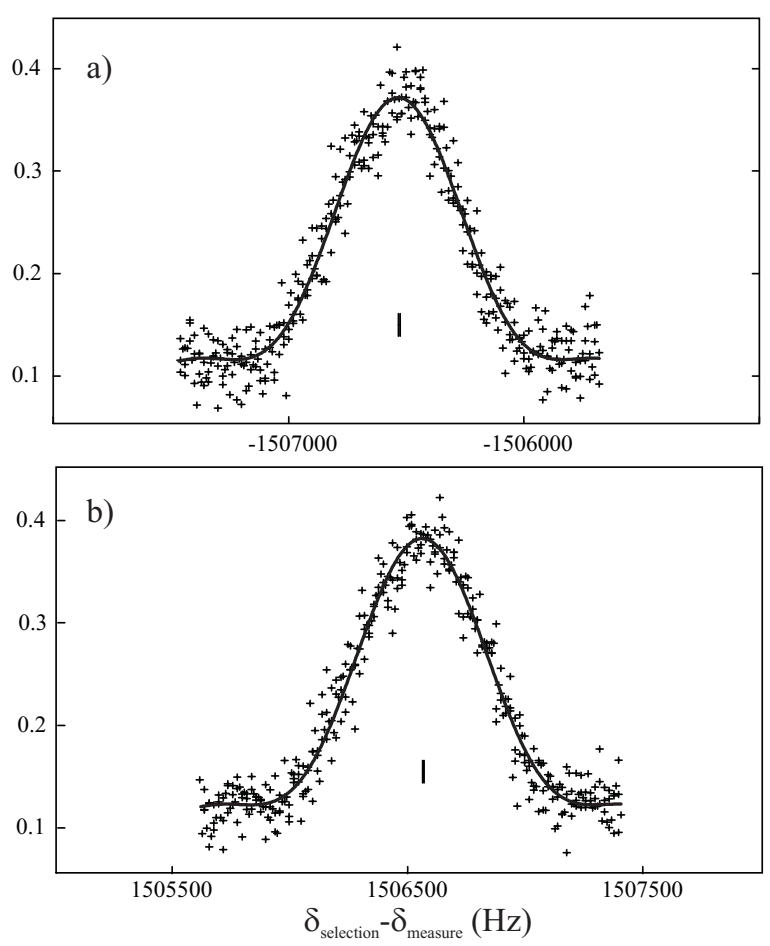

FIG. 3: Final velocity distribution for 50 Bloch oscillations, in both directions: a)Around $2 N v_{r}$ and b) Around $-2 N v_{r}$. The center of the final velocity distribution can be located with an uncertainty of $3 \mathrm{~Hz}$. The photon recoil frequency deduced from these two measurements is $15066.690(23) \mathrm{Hz}$.

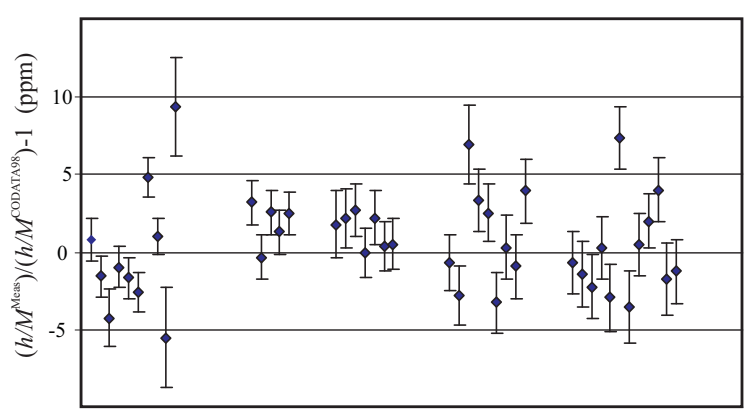

FIG. 4: Chronological display of the data taken for $N=50$ Bloch oscillations in both directions. The mean deviation from the expected value is $6.1(4.2) \times 10^{-7}$, with $\chi^{2}=99$.

scheme, or they are placed in a pure standing wave. In this case, the atoms oscillate around the same position at the frequency $m g / 2 \hbar k$ 24]. Furthermore, there is no significant displacement of the atom between the velocity selection and measurement, and thus, several systematic effects are reduced. On the other hand, a precise determination of the local gravity field is required to exploit this technique to the full. Finally we expect to increase the number of transferred recoils up to $N=500$, to ob- tain a determination of $v_{r}$ with an uncertainty better than $10^{-8}$, leading to a determination of $\alpha$ with an uncertainty of about $5 \mathrm{ppb}$, close to the more accurate value deduced from $(g-2)[11,12,16$

We thank A. Clairon, C. Salomon and J. Dalibard for valuable discussions. This experiment is supported in part by the Bureau National de Métrologie (contract 993009) and by the Région Ile de France (contract SESAME E1220).

[1] S. Chu, C. Cohen-Tannoudji, W.D. Phillips, Nobel lectures in physics Rev. Mod. Phys. 70, 685-741 (1998).

[2] G. Santarelli et al., Phys. Rev. Lett. 82, 4619 (1999).

[3] C.W. Oates, K.R. Vogel and J.L. Hall, Phys. Rev. Lett. 76, 2866 (1996).

[4] B. Taylor, Metrologia. 31, 181 (1994).

[5] C. Schwob, L. Jozefowski, B. de Beauvoir, L. Hilico, F. Nez, L. Julien, F. Biraben, O. Acef and A. Clairon, Phys. Rev. Lett. 82, 4960 (1999).

[6] Th. Udem, A. Huber, B. Gross, J. Reichert, M. Prevedelli, M. Weitz and T.W. Hänsch, Phys. Rev. Lett. 79, 2646 (1997).

[7] D.L. Farnham, R.S. Van Dyck and P.B. Schwinberg, Phys. Rev. Lett. 75, 3598 (1995).

[8] T. Beieret al., Phys. Rev. Lett. 88, 011603 (2002).

[9] M.P. Bradley, J.V. Porto, S. Rainville, J.K. Thompson and D.E. Pritchard, Phys. Rev. Lett. 83, 4510 (1999).

[10] W. Liu et al., Phys. Rev. Lett. 82, 711 (1999).

[11] T.Kinoshita, IEEE Trans. Instrum. Meas. 46, 108 (1997).

[12] R.S. Van Dyck, P.B. Schwinberg and H.G. Dehmelt, Phys. Rev. Lett. 59, 26 (1987).

[13] E. Krüger, W. Nistler and W. Weirauch, Metrologia 36, 147 (1999).

[14] J.Q. Shields, R.F. Dziuba and H.P. Layer, IEEE Trans. Instrum. Meas. 38, 249 (1989).

[15] A.M. Jeffery, R.E. Elmquist, L.H. Lee, J.Q. Shields and R.F. Dziuba, IEEE Trans. Instrum. Meas. 46, 264 (1997).

[16] P. Mohr and B. Taylor, Rev. Mod. Phys. 72, 351 (2000).

[17] J.L. Hall, C.J. Bord and K. Uehara, Phys. Rev. Lett. 37, 1339 (1976).

[18] C.J. Bordé, Phys. Lett. A 140, 10 (1989).

[19] B. Young, M. Kasevich and S. Chu, Atom Interferometry, edited by P.R Berman, (Academic, New York), 363 (1997).

[20] A. Wicht, J.M. Hensley, E. Sarajlic and S. Chu, Proceedings of the 6th Symposium on Frequency Standards and Metrology, eds. P. Gill (World Scientific, Singapore), pp 193-212 (2001).

[21] S. Gupta, K. Dieckmann, Z. Hadzibabic and D.E. Pritchard, Phys. Rev. Lett. 89, 140401-1 (2002).

[22] M. Weitz, B.C. Young, and S. Chu, Phys. Rev. Lett. 73, 2563 (1994).

[23] M. Ben Dahan, E. Peik, J. Reichel, Y. Castin and C. Salomon, Phys. Rev. Lett. 76, 4508 (1996).

[24] R. Battesti, P. Cladé, S. Guellati-Khélifa, C. Schwob, B. Grémaud, F. Nez, L. Julien and F. Biraben, J. Opt. B.: Quantum Semiclassical 5, S178-S182 (2003). 
[25] M. Kasevich, D.S. Weiss, E. Riis, K. Moler, S. Kasapi and S. Chu, Phys. Rev. Lett. 66, 2297 (1991).

[26] E. Peik, M. Ben Dahan, I. Bouchoule, Y. Castin and C. Salomon, Phys. Rev. A 55, 2989 (1997).
[27] A. Clairon, P. Laurent, G. Santarelli, S. Ghezali, S.N. Lea and M. Bahoura, IEEE Trans. Instrum. Meas. 44, 128 (1995). 